\title{
CHARGE STATE MEASUREMENTS OF SOLAR ENERGETIC PARTICLES OBSERVED WITH SAMPEX
}

\author{
G. M. Mason, ${ }^{1,2}$ J. E. Mazur, ${ }^{1}$ M. D. LoOper, ${ }^{3}$ and R. A. Mewaldt ${ }^{4}$ \\ Received 1995 January 23; accepted 1995 May 1
}

\begin{abstract}
We have measured the ionization states of major elements accelerated in two large solar energetic particle events by use of the geomagnetic cutoff technique. The observations were made in 1992 October/November using instrumentation on the $S A M P E X$ satellite, which is in an $82^{\circ}$ inclination low Earth orbit. We calibrate the geomagnetic cutoff rigidity by assuming that protons and He observed on SAMPEX are fully stripped. The mean charge states of the heavier elements are then determined from their measured cutoffs, along with this calibrated scale. Our results cover the energy range $\sim 0.5-5 \mathrm{MeV}$ nucleon $^{-1}$ and are in good agreement with earlier studies by Luhn et al. (1985) near $\sim 1 \mathrm{MeV}^{\text {nucleon }}{ }^{-1}$ in all cases except for Fe. We find a mean charge state of $11.04 \pm 0.22$ for $\mathrm{Fe}$, while the average from Luhn is $14.09 \pm 0.09$. The lower ionization state of $\mathrm{Fe}$ reported here appears to be consistent with the general range of values recently measured in solar wind $\mathrm{Fe}$ on the Ulysses deep space probe. Although the observational picture is still incomplete, overall the present results confirm and extend previous evidence that the solar energetic particles in large events are accelerated from the coronal or solar wind material.
\end{abstract}

Subject headings: cosmic rays - Sun: abundances - Sun: flares - Sun: particle emission

\section{INTRODUCTION}

Energetic particles emitted by the Sun carry important information about conditions and dynamic processes operating in and near the solar corona. The elemental and isotopic compositions give information about the speed population for the energetic particles, and the energy spectra (routinely extending to tens of $\mathrm{MeV}$ nucleon ${ }^{-1}$ and sometimes up to relativistic energies) carry signatures of the acceleration and transport processes operating in these events. While protons and helium nuclei are essentially fully stripped in the solar energetic particles, heavier elements are only partially stripped. Since the particle transport from the corona to Earth orbits is collisionless, the ionization states sampled at Earth carry direct information about the seed population and the conditions at the accelerating site.

Over the last several years, it has become evident that solar energetic particle events can be usefully separated into two classes: (1) "gradual" events, which are generally the largest type and are associated with long duration X-ray releases, coronal shocks, and coronal mass ejections; and (2) "impulsive" events, which are associated with small, compact $\mathrm{X}$-ray source emission, enrichments in electrons, ${ }^{3} \mathrm{He}$, and heavy elements (e.g., review by Lin 1987, and references therein). Ionization states of heavy ions have been measured in a number of large ("gradual") solar particle events (e.g., Sciambi et al. 1977; Hovestadt et al. 1981a; Adams et al. 1993), with the most complete survey reported by Luhn et al. (1985). These studies have shown that in general the ionization states of the elements $\mathrm{O}-\mathrm{Fe}$ are roughly consistent with equilibrium temperatures of a few times $10^{6} \mathrm{~K}$, although there are significant element-to-element differences. This observational picture has provided some of the strongest evidence that in large

\footnotetext{
${ }^{1}$ Department of Physics, University of Maryland, College Park, MD 20742.

${ }^{2}$ Institute for Physical Science and Technology University of Maryland.

${ }^{3}$ Aerospace Corporation, Los Angeles, CA 90009.

${ }^{4}$ California Institute of Technology, Pasadena, CA 91125.
}

(gradual) flares, the energetic particle seed population is the corona and not the site of the optical flare, which is much hotter $\left(>10^{7} \mathrm{~K}\right)$.

Because of the experimental difficulty of measuring the ionization states of solar energetic particles, the direct studies to date have been limited to energies below $\sim 1 \mathrm{MeV}^{\text {nucleon }}{ }^{-1}$, and all have used the same technique, namely electrostatic deflection of the particles followed by an energy measurement. In view of the importance of solar energetic particle ionization states, and in order to extend the energy range to higher values, we have used observations of the geomagnetic cutoffs of particles observed on the SAMPEX spacecraft to deduce the ionization states of solar energetic particles in two large particle events. This is a different technique from that used in the earlier studies, and in addition it allows extension of the measurements to higher energies.

Our deduced ionization states cover the range from $\sim 0.5$ to $5 \mathrm{MeV}$ nucleon ${ }^{-1}$ and are in good agreement with earlier studies by Luhn et al. (1985) in all cases except for Fe. We find a mean charge state of $11.04 \pm 0.22$ for $\mathrm{Fe}$, while the average from Luhn is $14.09 \pm 0.09$. As found in earlier studies, the ionization state values reported here for a range of elements are not consistent with any single equilibrium temperature in the solar corona. This may be due to the operation of other mechanisms near the flare, such as ionization by X-rays (Mullan \& Waldron 1986). However, if the particles are accelerated at coronal heights or above, then the solar wind is the seed population, not the material at the optical flare site. In this case, the solar energetic particle ionization states should be compared with those of the solar wind. While a direct comparison is not yet possible, the ionization states we observe appear to be consistent with the range of ionization states measured in the solar wind surveys by instruments on the Ulysses deep space probe (Ipavich et al. 1992; von Steiger \& Geiss 1995).

\section{INSTRUMENTATION}

The observations presented here were made with two of the instruments on the SAMPEX (Explorer 68) satellite, which was 
launched into a $675 \times 512 \mathrm{~km} 82^{\circ}$ inclination Earth orbit on 1992 July 3 (for an overview of the mission, see Baker et al. 1993). Thus, the spacecraft orbit passes over the polar caps where the solar energetic particles have free access, and then as it moves to lower magnetic latitudes, the particle fluxes are cut off by the geomagnetic field at locations dependent on their gyroradii. The spacecraft telemetry includes the particle arrival times and the spacecraft location, which makes it possible to analyze the details of the particle cutoffs in groundbased data processing.

The heavy ion data reported here were obtained with the University of Maryland Low Energy Ion Composition Analyzer (LICA), which uses a novel time-of-flight technique in which the ion flight times are measured over a $0.5 \mathrm{~m}$ flight path, and their residual energies are measured in standard silicon solid state detectors. These measurements determine the ion velocity $v$ and kinetic energy $m v^{2} / 2$, which makes it possible to solve for the mass $m$. The technique allows very low energy thresholds along with large collection power, making it ideal for studies of solar particle events. LICA determines only the mass of the incident ions, not their nuclear charge $Z$; however, for isotopes of mass below $\mathrm{Ni}$, there are no significant ambiguities for isobars in material of solar system abundances, and so nuclear species can be identified as well. A complete description of the LICA instrument has been published by Mason et al. (1993).

Proton measurements reported here were carried out with the California Institute of Technology/Goddard Space Flight Center Proton Electron Telescope (PET), which identifies protons by the well-known $d E / d x$ versus residual energy method. The PET instrument has been described in detail by Cook et al. (1993). Both PET and LICA have identical viewing directions, an important consideration in the analysis below, which combines particle cutoff information from both instruments.

\section{OBSERVATIONS}

\subsection{Selection of Time Period}

Figure 1 shows fluxes of 20-27 MeV protons and of several low-energy $O$ fluxes during the period 1992 October 30November 7 during portions of the SAMPEX orbit when the spacecraft was at invariant latitudes $\Lambda>70^{\circ}$. At such high latitudes, interplanetary ions can reach the satellite with negligible perturbation from the geomagnetic field. Two solar flares are associated with the energetic particle increases: a $2 \mathrm{~N}$ flare at S26W63 that started at 17:02 UT on October 30, and a 2B flare at S23W90 that started at 03:14 UT on November 2. Both flares originated in the same active region on the Sun, and both were followed by storm sudden commencements (SSC) that are indicated on the figure (flares and SSC identifications were obtained from Solar Geophys. Data, Part II. 1993, Nos. 584 and 585). In addition, an interplanetary shock (S) associated flux increase can be seen around 08:00 UT on November 3. The location of the flares in the western hemisphere "well-connected" region is consistent with the fast rise times seen for the photon fluxes, and both events are typical of the large, "gradual" events associated with shock acceleration in or near the corona (Mason, Gloeckler, \& Hovestadt 1994; Cane, Reames, \& Von Rosenvinge 1988; Gosling 1993). During the current declining phase of the solar activity cycle, these two events were the largest solar particle events observed by $S A M P E X$ from launch through 1995 May.

While the 20-27 MeV protons show two distinct particle events for this period, note that the situation is not as clear at lower energies. The LICA instrument was off during the initial rise phase of the flare, and so heavy ion data coverage does not begin until about 18:00 UT on October 31. However, because of velocity dispersion, the lower energy fluxes had not reached their maximum intensity even by this time. Examining the oxygen fluxes in Figure 1 at $0.55,1.50$, and $4.25 \mathrm{Mev}$

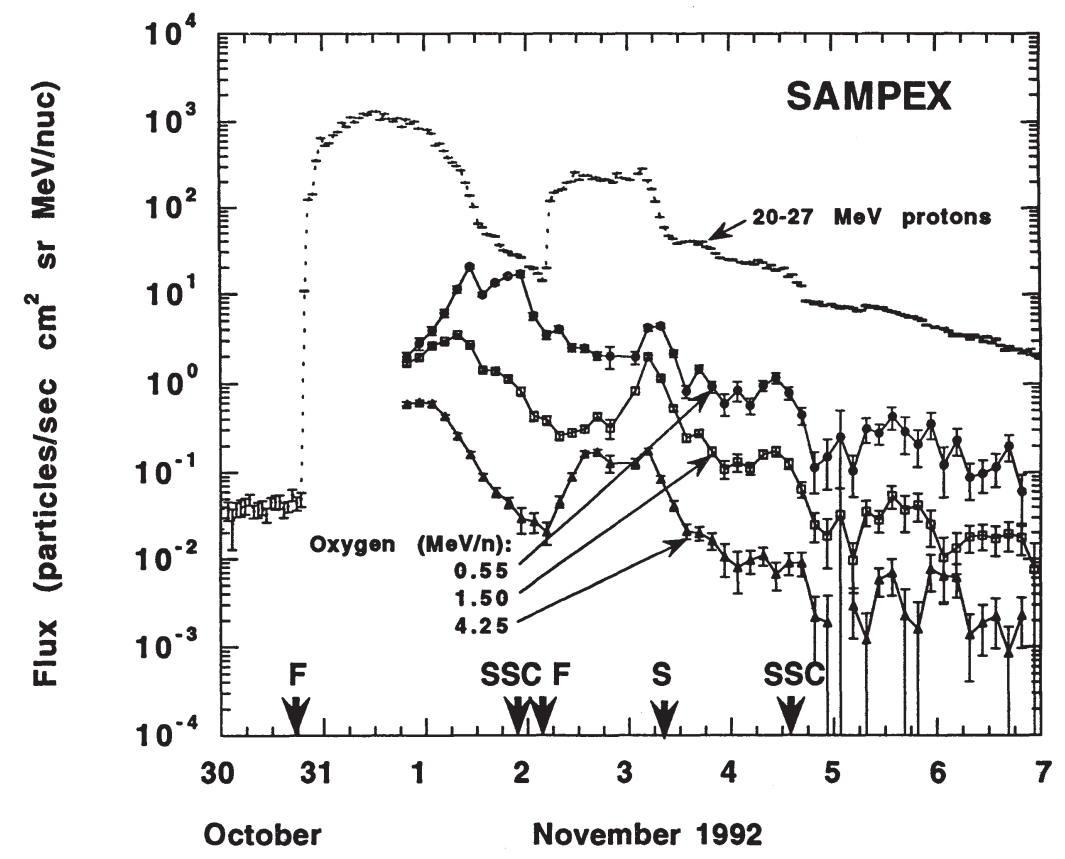

FIG. 1.-Time history of selected fluxes during the period 1992 October 30-November 7 measured at invariant latitudes $>70^{\circ}$. Arrows marked F denote times of

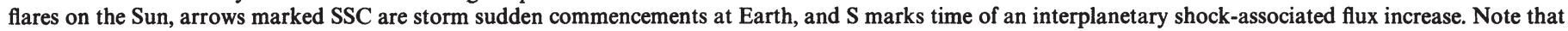
while there were two distinct events observed at high energies, the lower energy data appear to be dominated primarily by the first event. 
nucleon $^{-1}$, it can be seen that at the two lower energies the second event did not produce large enough fluxes of particles to stand out. The main feature seen after the initial maximum in the low-energy fluxes is the relatively short-lived increase associated with a shock passage around 08:00 UT on November 3. However, it is apparent from the figure that most of the low energy particles are associated with the October 30 event or the shock passage.

In order to explore this question further, Figure 2 shows differential energy spectra averaged over the entire period October 30 18:00 UT-November 6 00:00 UT. We note that the $\mathrm{Fe} / \mathrm{O}$ ratio near $1 \mathrm{Mev}$ nucleon ${ }^{-1}=0.41 \pm 0.03$. This is considerably higher than values $0.031 \pm 0.007$ and 0.071 \pm 0.006 obtained for the two events seen at higher energies from $\sim 20$ to $70 \mathrm{MeV}$ nucleon ${ }^{-1}$ by Selesnick et al. (1993). The low-energy spectra in Figure 2 are well represented by power laws in kinetic energy per nucleon and show no signs of a break or other structure that would give evidence that at low energies we are dealing with two distinctly different particle populations. For these reasons, along with the need to increase statistical accuracy in the measurements, the analysis below sums over the entire period of the two events from 18:00 UT on October 31 through 00:00 UT on November 6.

\subsection{Determination of Cutoffs}

The technique for deducing the particle ionization states in this study makes use of the fact that in the geomagnetic field, access of particles to a spacecraft in low Earth orbit is limited by the details of the particle's trajectory through the field. In steady state conditions, this trajectory depends only on the particle gyroradius and the sign of particle charge. Usually this condition is formulated by stating that the particle must have a magnetic rigidity (momentum per unit charge) greater than the so-called cutoff rigidity, $R_{C}$, which is the minimum rigidity for which a particle from interplanetary space can reach any particular point in the spacecraft's orbit for a given look direction (e.g., Rossi \& Olbert 1970). Then, for particles just at the cutoff rigidity,

$$
R_{C}=\frac{m v}{Q e},
$$

so if the cutoff rigidity is known, the charge state $Q$ can be obtained immediately from

$$
Q=\frac{m v}{R_{C} e} .
$$

The analysis that follows therefore has two main stages: first, to find the cutoff location (in invariant latitude) of the heavy ion species whose charges we wish to measure, and second, to calibrate the cutoff location (invariant latitude) scale in terms of magnetic rigidities. When this is done, the mean charge state is determined immediately from equation (2), since the instrument measures the mass $m$ and velocity $v$ of all the ions.

In principle, it might be possible to calculate the cutoff rigidities for our orbit using the properties of the geomagnetic field and external sources such as Earth's ring current (e.g., Schulz \& McNab 1987). However, especially at the relatively low energies studied here, this is not practical since the field models are not sufficiently precise and since external current sources have a significant effect on the cutoffs. Figure 3 shows the behavior of the cutoffs during the 1992 October/November time period (hereafter, when the word cutoff is used, we refer to the cutoff invariant latitude). The dots plotted at the upper portion of the panel show the magnetic invariant latitude (McIlwain 1961) of each oxygen nucleus of $>0.5 \mathrm{MeV}$ nucleon $^{-1}$ (the hatched boxes at the top of the figure mark periods when the LICA instrument was off). It can be clearly seen that the low-energy oxygen can reach lower latitudes at some times than at others. This behavior is followed closely by the 20-27 MeV proton cutoffs, which are also shown in the figure (displaced downward by $5^{\circ}$ for clarity), as well as other higher energy ions (Leske et al. 1995).

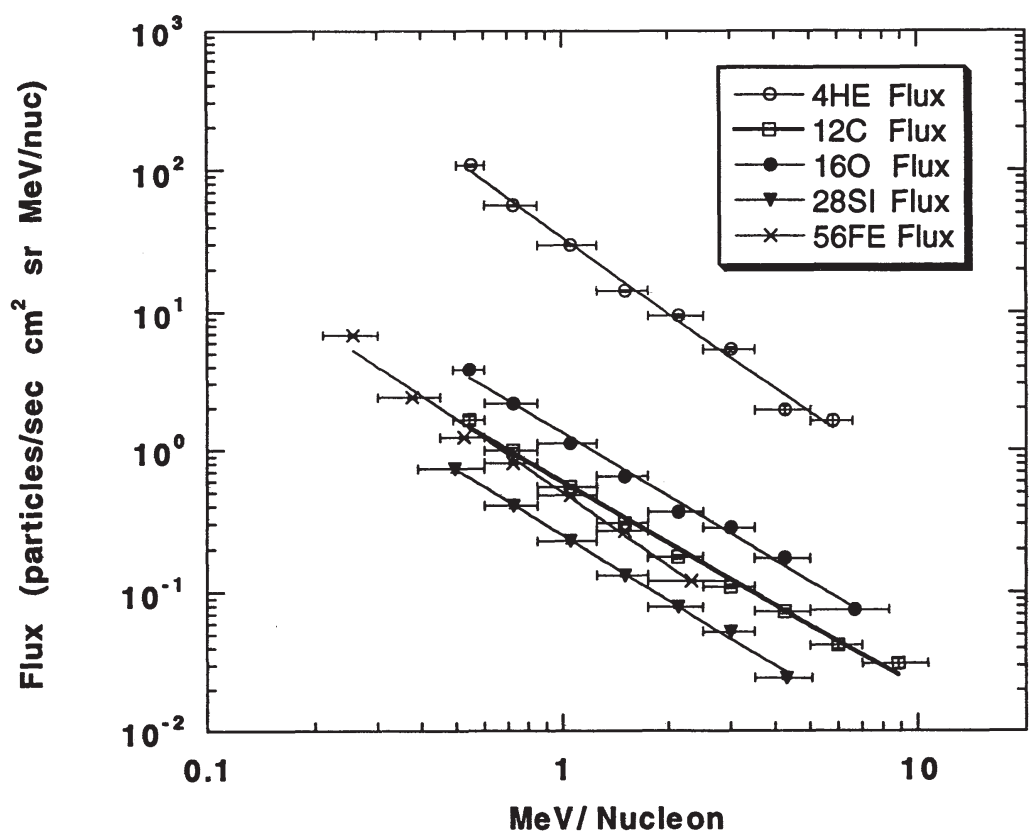

FIG. 2.-Kinetic energy per nucleon spectra of selected heavy ions measured at invariant latitudes $>70^{\circ}$ during the period 1992 October $3018: 00$ UT-1992 November 600:00 UT. 


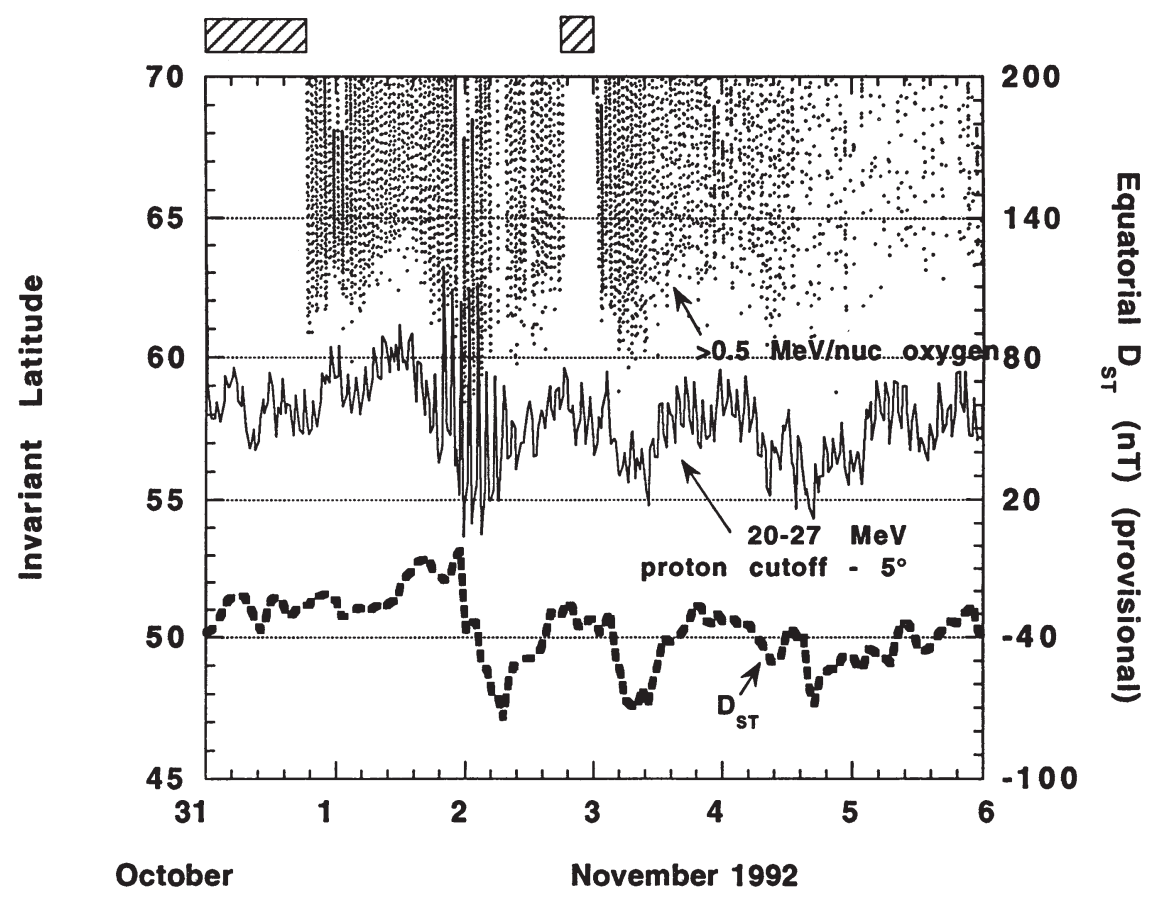

FIG. 3.-Display of the time variations of geomagnetic cutoff during the particle events: hatched boxes at top show times when LICA was off; dots show the arrival invariant latitude for individual ions of $>0.5 \mathrm{MeV}$ nucleon ${ }^{-1}$ oxygen; the thin line is the cutoff determined by $20-27 \mathrm{MeV}$ protons, displaced by $5^{\circ}$ for clarity; and the dashed line is the preliminary $D_{\mathrm{ST}}$ (scale is on right axis). Note the similarity between the energetic particle cutoff variations and the time dependence of $D_{\mathrm{ST}}$.

The proton cutoffs are more accurately determined than the oxygen cutoffs since the count statistics are much higher. Superposed on the overall structure of the proton cutoff in Figure 3 is a fine structure which is due to cutoff differences between the north versus south polar cap and dayside versus nightside (e.g., Evans \& Stone 1969; Hovestadt \& Meyer 1969; Van Allen, Fennell, \& Ness 1971; Cooper, Guzik, \& Werfel 1993). Also shown in the figure is the provisional $D_{\mathrm{ST}}$ (disturbance storm time) (kindly supplied by Dr. M. Sugiura at Tokai University), which is a measure of the perturbation of Earth's equatorial magnetic field due to changes in the ring current. There is a clear similarity between the $D_{\mathrm{ST}}$ time variations and the observed cutoff, which indicates that the changes in cutoff that we observe are influenced by the dynamical changes in the ring current (Leske et al. 1995). This has also been observed at higher energies in other events by Flückiger, Smart, \& Shea (1986).

Because of the time variations in the cutoff, it is necessary to introduce corrections to the cutoff value observed during each entry into or exit from the polar cap so that a meaningful average can be formed over the entire period. The heavy ion data from LICA are not suitable for this purpose because counting statistics are not sufficient (e.g., see Fig. 3). The 20-27 $\mathrm{MeV}$ proton count rate from the PET sensor, however, has excellent count statistics and can be used to determine the cutoffs for each polar pass. Figure 4 shows an example of the PET proton data for a pass on 1992 November 3, when fluxes were well below their peak values. On the figure, "entry" and "exit" times are marked where the proton count rate fell to one-half of the average value over the polar cap. These times were identified for all polar passes, yielding a list of times when the spacecraft passed through a cutoff. Then, each of these times was converted to an invariant latitude (A) value by referring to the satellite position at the time of the cutoff. The average invariant latitude and dispersion for $20-27 \mathrm{MeV}$ protons were $\Lambda_{0}=62.07 \pm 1.02$.

In order to locate the average cutoffs for each heavy ion species and energy, the LICA fluxes for each polar pass were calculated and binned according to an adjusted invariant latitude $\left(\Lambda_{\mathrm{adj}}\right)$ calculated from the observed proton cutoff from that pass $\left(\Lambda_{i}\right)$ using a simple multiplicative correction:

$$
\Lambda_{\mathrm{adj}}=\Lambda\left(\frac{\Lambda_{0}}{\Lambda_{i}}\right) .
$$

For the first half of a polar pass, the observed cutoff $\left(\Lambda_{i}\right)$ at the entry to the polar cap was used, and for the second half the observed cutoff $\left(\Lambda_{i+1}\right)$ for the exit was used in equation (3). The algorithm leaves some ambiguity concerning the latitude adjustment near the middle of the polar pass, but this is not a significant problem since the critical region of the data is near the cutoffs. Leske et al. (1995) have corrected for these latitude variations by subtraction; there is little difference between these two methods since the overall correction is centered around the average $\Lambda$ value, and the dispersion is small.

Figure 5 shows an example of $0.85-1.25 \mathrm{MeV}$ nucleon $^{-1}$ fluxes for three species plotted versus adjusted invariant latitude in 0.2 bins. In order to reduce statistical fluctuations, the fluxes were then "smoothed" by taking a running average over $\pm 1^{\circ}$ invariant latitude around each point. This bin width was sufficiently narrow to follow the shape of the flux decreases accurately near cutoff. From the three species plotted in the figure, it is immediately apparent that $\mathrm{Fe}$ has a significantly lower charge-to-mass ratio than those of $\mathrm{O}$ or $\mathrm{He}$, since it clearly penetrates to lower latitudes even though it has the same velocity as the $\mathrm{O}$ and $\mathrm{He}$.

The cutoff value for each flux trace such as those in Figure 5 was then defined as the invariant latitude at which the flux 


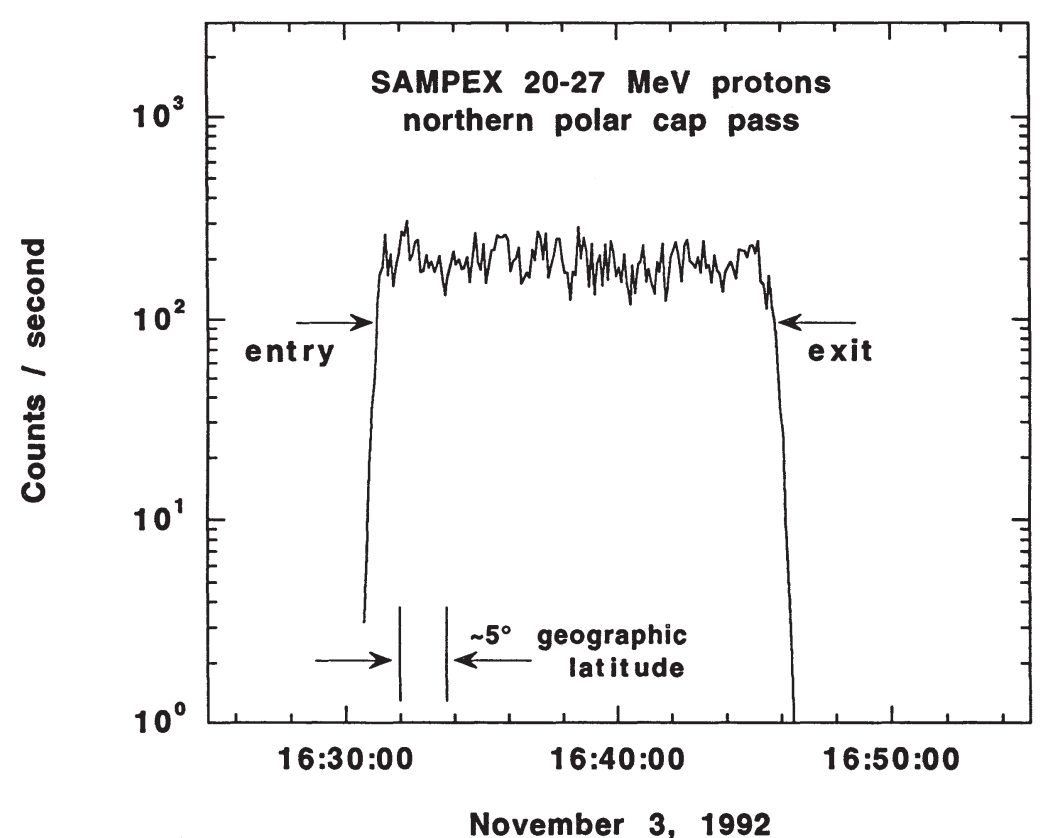

Fig. 4.-Example of the data used to determine the cutoff using $20-27 \mathrm{MeV}$ protons. Polar cap entry and exit are defined as those times when count rates cross a threshold value of $50 \%$ of the average value over the polar cap.

reached 0.50 of its average value over the polar cap at invariant latitudes $\Lambda>70^{\circ}$. Because of the running average procedure used to smooth the flux versus latitude plots, and because of the variations seen in the fluxes even at latitudes $>65^{\circ}-70^{\circ}$ (see Fig. 5), it is difficult to estimate directly uncertainties in cutoff latitudes deduced by this procedure. The technique used was to assign a nominal $10 \%$ uncertainty to the polar cap flux value (or its statistical uncertainty, if it was larger than 10\%) and to recalculate the cutoff latitude with these values. This has the effect of causing larger uncertainties in cutoffs at low energies, since the cutoffs are less sharp there, and a $10 \%$ change in cutoff flux level corresponds to a larger change in invariant latitude than when the cutoff is a sharper function of latitude as is the case at higher energies. We also examined the effect of changing the cutoff threshold from 0.50 of the polar cap value to some lower fraction (e.g., 0.30 or 0.10 ). Of course, the deduced cutoff values for each species and energy were lower with the lower thresholds; however, the eventually deduced

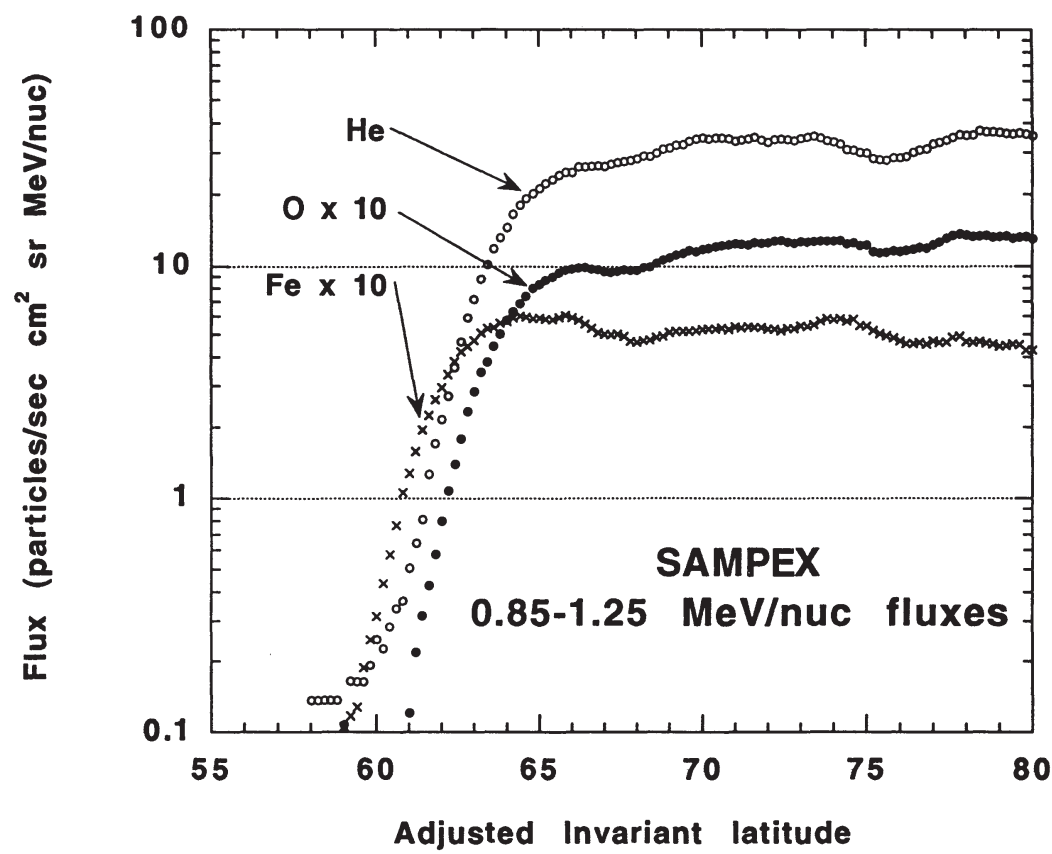

Fig. 5.-Fluxes of $0.85-1.25 \mathrm{MeV}$ nucleon ${ }^{-1} \mathrm{He}, \mathrm{O}$, and $\mathrm{Fe}$ averaged over the entire time period and plotted as a function of adjusted invariant latitude. The $\mathrm{O}$ and $\mathrm{Fe}$ fluxes were multiplied by 10 . Note that $\mathrm{Fe}$ penetrates to a much lower invariant latitude than He or $\mathrm{O}$. 
charge states were not affected by such changes, within the stated uncertainties. It was decided to use the 0.50 cutoff criterion since it gave better statistical accuracy.

The cutoffs for the major ion species are plotted as a function of kinetic energy per nucleon in Figure 6. The two proton points are from the PET 20-27 MeV and 65-85 MeV channels and were determined by computing the PET proton fluxes as functions of adjusted invariant latitude in the same manner used for heavy ions. In addition to the points shown in the figure, cutoffs were calculated for minor ion species $\mathrm{N}, \mathrm{Ne}, \mathrm{Mg}$, $\mathrm{Si}, \mathrm{S}$, and $\mathrm{Ca}$. The minor ion species cutoffs were generally similar to those for the major species but with more scatter in the points.

If all the ion species had the same mass-to-charge ratio, then at the same kinetic energy per nucleon they would have the same magnetic rigidity and would therefore have the same cutoffs. As expected, the protons are well separated from the other species. However, the heavier ion species show a systematic trend wherein heavier ions at the same energy per nucleon penetrate to lower latitudes than lighter ones. This is the trend predicted if the heavier ions have larger mass-to-charge ratios than the lighter ones, i.e., if they are not as fully stripped of their electrons.

\section{DERIVATION OF IONIZATION STATES}

In order to use equation (2) to determine the charge of each ion, it is necessary to calibrate the invariant latitude cutoff scale so that each latitude point can be associated with a magnetic cutoff rigidity $R_{c}$. This can be done by introducing the additional assumption that the protons and $\mathrm{He}$ are both fully stripped of their orbital electrons. Then the magnetic rigidity can be calculated from equation (1), and the results are shown in Figure 7. Note the excellent agreement between the 20-27 $\mathrm{MeV}$ proton point and the $\mathrm{He}$ points at 4.3 and $5.8 \mathrm{MeV}$ nucleon $^{-1}$, whose cutoffs are nearly identical even though the ion velocities differ by a factor of $\sim 2$. In order to derive a smooth rigidity versus invariant latitude curve, we fitted a third-order polynomial through the points and used it for our calibrated cutoff curve. A polynomial was chosen in preference to, e.g., an exponential in order to better fit the bend in the data above $64^{\circ}$. Comparing Figure 7 to Figure 6 , note that the proton and helium data, and therefore our calibration curve for converting cutoff latitude to rigidity, cover the entire cutoff range of the LICA heavy ion data, thereby removing any need to extrapolate the function beyond the range of our measured cutoffs. This removes a potential source of systematic error, since the shape of the cutoff curve at these energies is only empirically determined.

The cutoff values shown in Figure 7 are compared with magnetic field model calculations in Figure 8. The heavy dashed line shows the cutoff in the dipole field approximation. The groups of points above the dipole field cutoffs are sample individual cutoff values taken from the world map of vertical cutoffs calculated numerically by Shea \& Smart (1983) using the tenth-degree International Geomagnetic Reference Field. Each dot shows a cutoff value at a particular spot in the $S A M P E X$ orbit, with points separated by $6 \mathrm{~s}$ of orbital motion. The vertical cutoffs from a series of 16 consecutive orbits of the spacecraft during the particle event are shown, and the range in cutoff rigidities for a given invariant latitude is due to the differing longitudes of the spacecraft during this period. It is clear that the solar energetic particles penetrate to much lower invariant latitude than is expected on the basis of the models and that a smooth line through the LICA and PET values also has a different slope from the calculated cutoffs. The calculations shown in Figure 8 are for Earth's field only, i.e., they do not include effects due to outside sources such as the ring current. However, comparing this figure with Figure 3, it is clear that even late in the event, when the fluctuations in $D_{\mathrm{ST}}$ are relatively small, low-energy oxygen penetrates well below

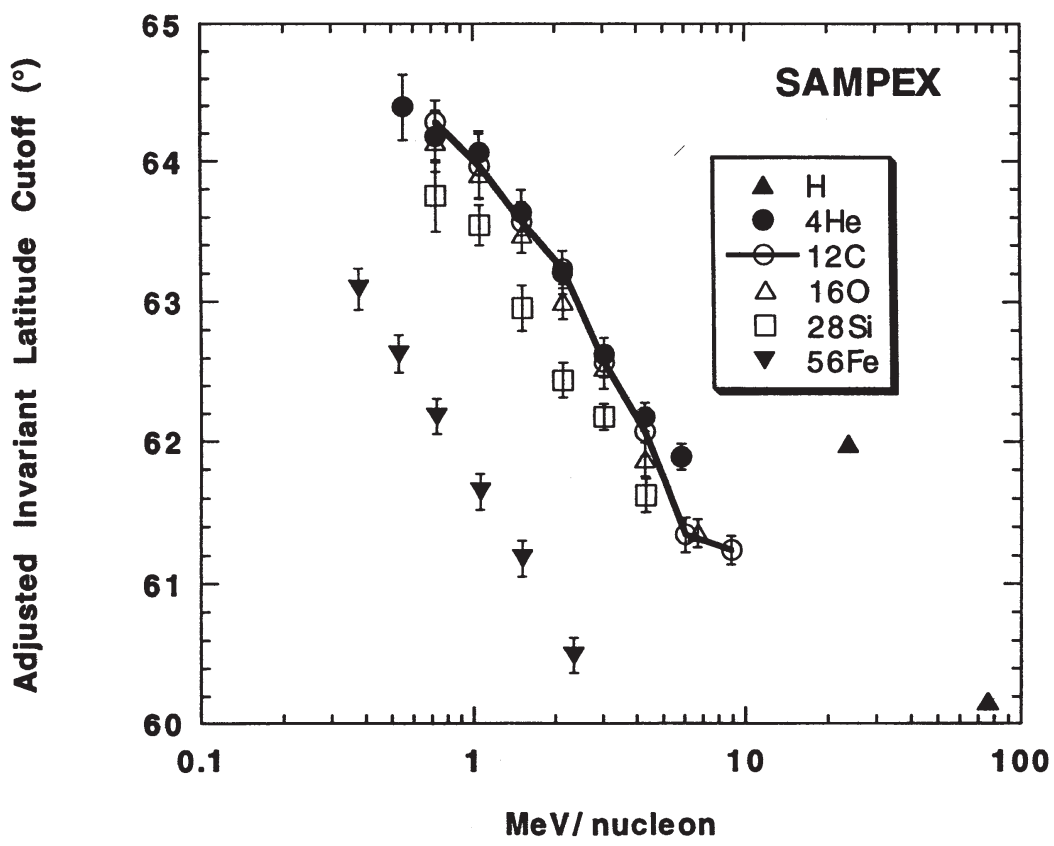

FIG. 6. - Cutoffs of low-energy ions measured during the period 1992 October 30 18:00 UT to November 7 00:00 UT. If all the ions had the same mass-to-charge

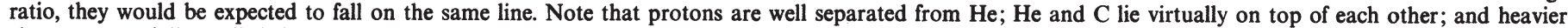
elements, especially $\mathrm{Fe}$, lie below $\mathrm{He}$ and $\mathrm{C}$. $\mathrm{C}$ points are connected by a heavy line to guide the eye. 


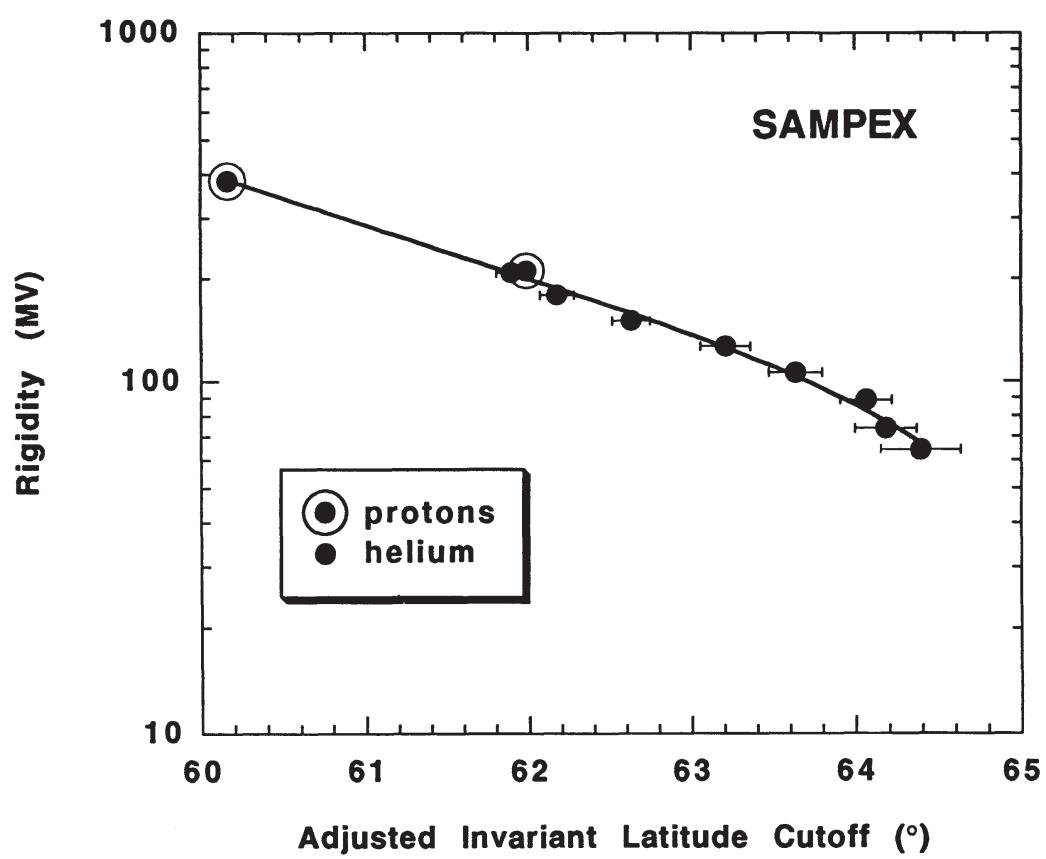

Fig. 7. - Calibration of the rigidity cutoff vs. invariant latitude scale carried out by assuming that both $\mathrm{H}$ and $\mathrm{He}$ are fully stripped. Points with large circles are determined from $20-27 \mathrm{MeV}$ and $65-85 \mathrm{MeV}$ protons. The smooth line is a third-order polynomial fit to the data used to derive cutoff rigidities from the observed cutoff latitudes for other species.

$70^{\circ}$ invariant latitude, which would not be expected on the basis of the field model calculations.

Using the polynomial functional fit in Figure 7, we used equation (1) to deduce charge states for the heavy ion species $\mathrm{C}, \mathrm{O}, \mathrm{Si}$, and $\mathrm{Fe}$; these are shown as functions of kinetic energy in Figure 9. Helium is also included, but since its ionization state was assumed to be exactly 2 , the points show only the effects of the fluctuation of the He cutoff values around the polynomial curve in Figure 7. Note that the deduced ioniza- tion states for each element are all essentially independent of energy over their entire range. The uncertainties shown in the figure are largest at low energies, which is somewhat surprising since the statistical accuracy is greatest there. The reason is the variation of the shape of the flux versus latitude curves (e.g., Fig. 5), which becomes progressively less sharp as energy decreases. Table 1 lists the weighted least-squares fits to the charge states for all the elements studied in the flare. For the less abundant elements, there was also no indication of an

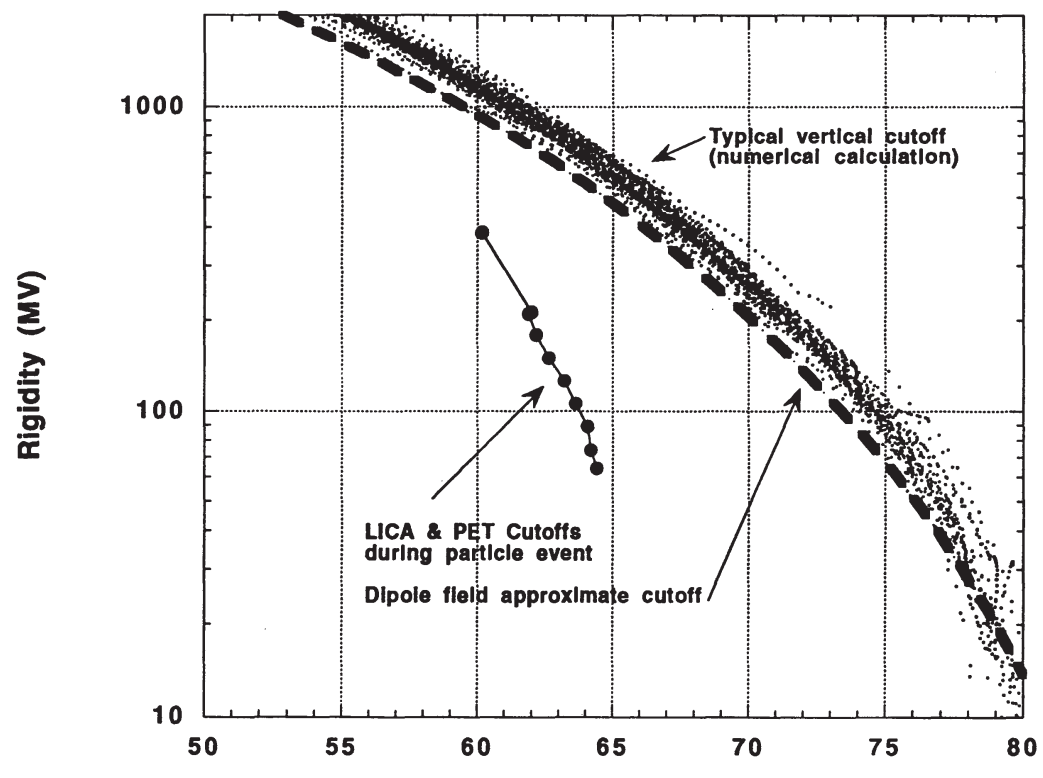

$\Lambda$, Invariant Latitude

FIG. 8. - Comparison of the cutoffs deduced in this study with nominal vertical cutoffs from a dipole model (dashed line) and numerical integrations of a multipole field model (dots; Shea \& Smart 1983). Dots show vertical cutoffs for several spacecraft orbits; the range of values is due to the varying orbital longitude. 


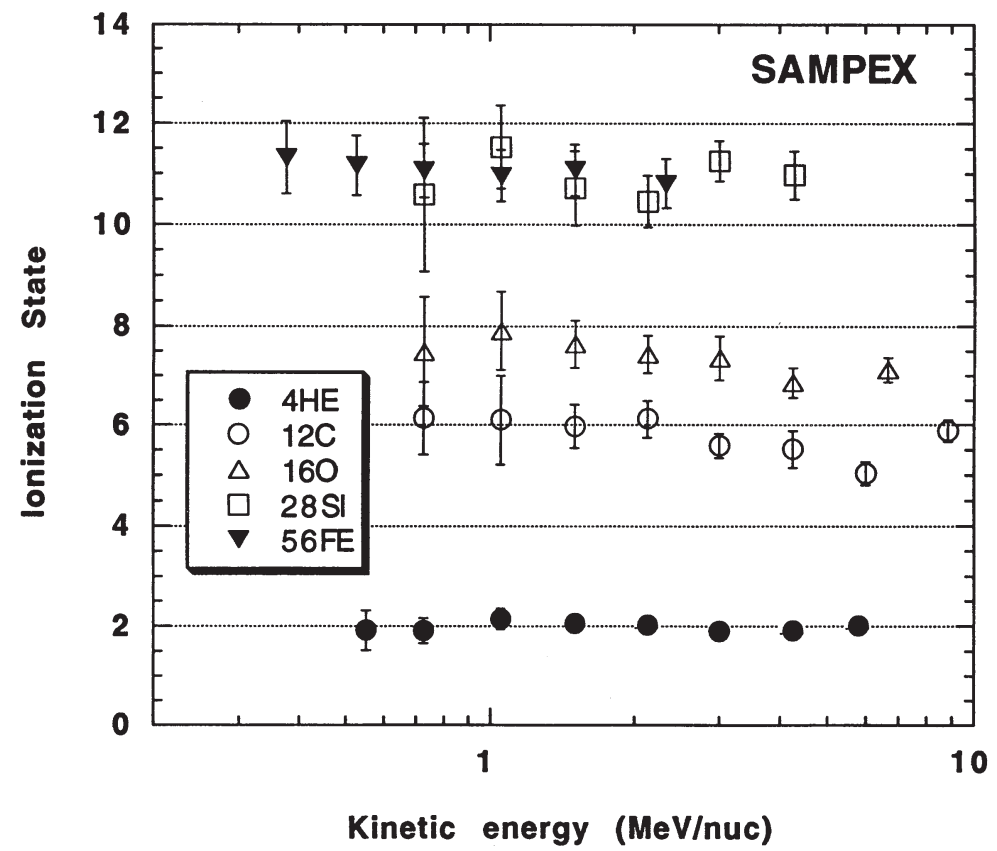

FIG. 9.-Deduced ionization states for major elements determined from geomagnetic cutoffs and the cutoff calibration shown in Fig. 7. Uncertainties are larger at low energies even though statistical accuracy is better there, because the cutoffs are less sharp at low energy. Notice the lack of any systematic energy dependence in the ionization states.

energy dependence in the ionization state; however, the statistical fluctuations were larger than those shown in Figure 9.

\section{DISCUSSION}

\subsection{Comparison with Previous Work}

In Figure 10 we compare the mean ionization states derived here with the average values from the work of Luhn et al. (1985), who surveyed 12 solar energetic particle events during 1978-1979. Luhn's survey was carried out near $1 \mathrm{MeV}$ nucleon ${ }^{-1}$ using an instrument that combined an electrostatic analyzer with a $d E / d x$ versus $E$ telescope. The uncertainty shown for the data points in the figure is a nominal 5\% systematic error estimated by Luhn, which we believe is the appropriate uncertainty to quote when comparing values taken with a different instrument. The variation among the flares in the Luhn survey was small, and so the average charge state uncertainties were also very small (e.g., 0.02 charge units for $\mathrm{C}$ and 0.09 charge units for $\mathrm{Fe}$ ). The agreement between the two mea-

TABLE 1

Average Ionization States and Implied Temperatures: 1992 OCTOBER 30-NOVEMBER 6

\begin{tabular}{lcc}
\hline \hline Element & $\begin{array}{c}\text { Mean } \\
\text { Charge State }\end{array}$ & $\begin{array}{c}\text { Equilibrium } \\
\text { Temperature }\left(\times 10^{6} \mathrm{~K}\right)\end{array}$ \\
\hline${ }^{\mathrm{He}} \ldots \ldots$ & $2.00 \pm 0.04^{\mathrm{a}}$ & $>0.10$ \\
${ }^{12} \mathrm{C} \ldots \ldots$ & $5.63 \pm 0.11$ & $1.48 \pm 0.10$ \\
${ }^{14} \mathrm{~N} \ldots$. & $6.52 \pm 0.16$ & $2.16 \pm 0.23$ \\
${ }^{16} \mathrm{O} \ldots$. & $7.21 \pm 0.15$ & $2.70 \pm 0.20$ \\
${ }^{20} \mathrm{Ne} \ldots$ & $9.38 \pm 0.16$ & $5.65 \pm 0.54$ \\
${ }^{24} \mathrm{Mg} \ldots$ & $10.62 \pm 0.55$ & $6.37 \pm 2.31$ \\
${ }^{28} \mathrm{Si} \ldots$. & $10.98 \pm 0.23$ & $1.88 \pm 0.07$ \\
${ }^{32} \mathrm{~S} \ldots \ldots$ & $11.91 \pm 0.25$ & $2.37 \pm 0.08$ \\
${ }^{40} \mathrm{Ca} \ldots$ & $11.83 \pm 0.21$ & $2.26 \pm 0.12$ \\
${ }^{56} \mathrm{Fe} \ldots$. & $11.04 \pm 0.22$ & $1.47 \pm 0.04$ \\
\hline
\end{tabular}

a Normalization. surements is excellent for the range $\mathrm{C}$ through $\mathrm{Si}$. For $\mathrm{S}$, the SAMPEX measurement is somewhat higher than the Luhn result, although the $1 \sigma$ errors nearly overlap. There was no $\mathrm{Ca}$ ionization state reported by Luhn to which we can compare the SAMPEX result. For Fe, however, the mean charge state reported here, $11.04 \pm 0.22$, is well below the Luhn value of $14.9 \pm 0.8$ using the $5 \%$ systematic error estimate, or $14.9 \pm 0.1$ if only statistical errors are considered.

A more detailed comparison of the Fe charge states in the Luhn et al. (1985) study with the present result is shown in Figure 11. The figure shows the ionization state from each of the 12 events in the Luhn study. Only event 5 in the survey had a mean $\mathrm{Fe}$ charge state consistent with the result presented here, and that event had very low statistical significance as can be seen from the large quoted uncertainty. Since the events studied here are not in the Luhn study, it is possible that event-to-event variations are the cause of the discrepancy shown in the figure. If that is the case, then the current result shows that these variations are considerably larger than would be assumed from the earlier survey. Given the large number of events in the Luhn survey and the fact that all the flares with high statistical accuracy have Fe charge states between $\sim 14$ and 16, we are inclined to regard the discrepancy as real and not due to statistical or event-to-event fluctuations.

When we search for other possible causes of this discrepancy in the Fe charge states, we note that earlier we assumed that He was fully stripped in order to derive the rigidity versus invariant latitude relation. However, interstellar pick-up $\mathrm{He}^{+}$ has been detected in association with solar energetic particle events, generally at a level of $\sim 10 \%$ or less over the range 0.3-2.4 MeV nucleon ${ }^{-1}$ (Gloeckler et al. 1981; Hovestadt et al. 1981b; Möbius et al. 1985; Gloeckler et al. 1994). For $10 \%$ $\mathrm{He}^{+}$, the mean charge state of $\mathrm{He}$ is 1.9 instead of the 2.0 assumed here; that in turn would raise the rigidity values in the curve shown in Figure 7. Propagated through the rest of the analysis, this would have the effect of raising the deduced rigid- 


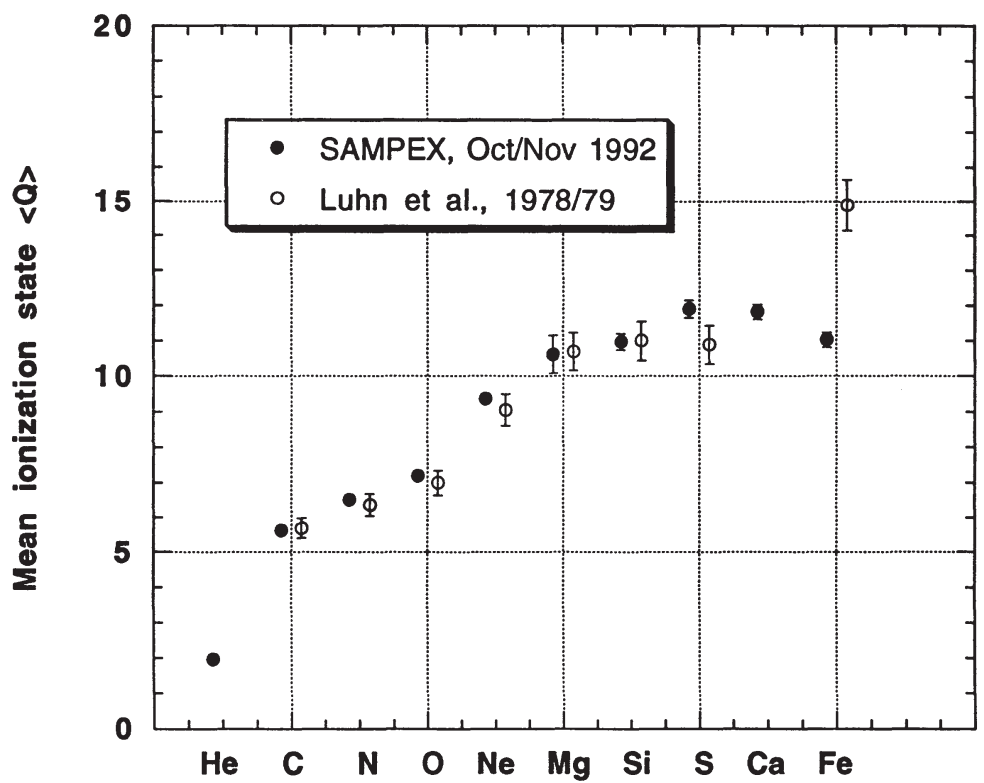

Fig. 10.-Comparison of the present results with the study of Luhn et al. (1985) who used an electrostatic analyzer on the interplanetary ISEE 3 spacecraft. The energy range of Luhn et al. is near $\sim 1 \mathrm{MeV}$ nucleon ${ }^{-1}$ and is an average over 12 separate particle events. Variations among the different events in Luhn's study were small. Note the excellent agreement except for the case of $\mathrm{Fe}$.

ity of the Fe nuclei, which in turn implies a lower ionization state. So if we assume any $\mathrm{He}^{+}$in the current solar energetic particle event, it would have the effect of increasing the discrepancy with Luhn et al. (1985).

\subsection{Implied Ionization Temperature}

As in previous studies, we compare the ionization states observed here with those expected for an equilibrium temperature in the solar corona. Table 1 and Figure 12 show the implied temperatures from our charge state measurements using the recent compilation of Arnaud \& Rothenflug (1985).
Except for $\mathrm{Ne}$ and $\mathrm{Mg}$, the elements are all consistent with a range between $\sim 1.5$ and $2.7 \times 10^{6} \mathrm{~K}$. This same trend was also pointed out by Luhn et al. (1985), which is expected given the agreement shown in Figure 11. The implied equilibrium temperature for $\mathrm{Fe}$ is at the lower end of this range (for the Luhn et al. 1985 ionization state, the implied temperature is about $22 \times 10^{6} \mathrm{~K}$, near the middle of the range).

\subsection{Implications for Particle Acceleration}

Because of essentially collisionless transport of the solar wind from a few solar radii to $1 \mathrm{AU}$ (e.g., Hundhausen, Gilbert,

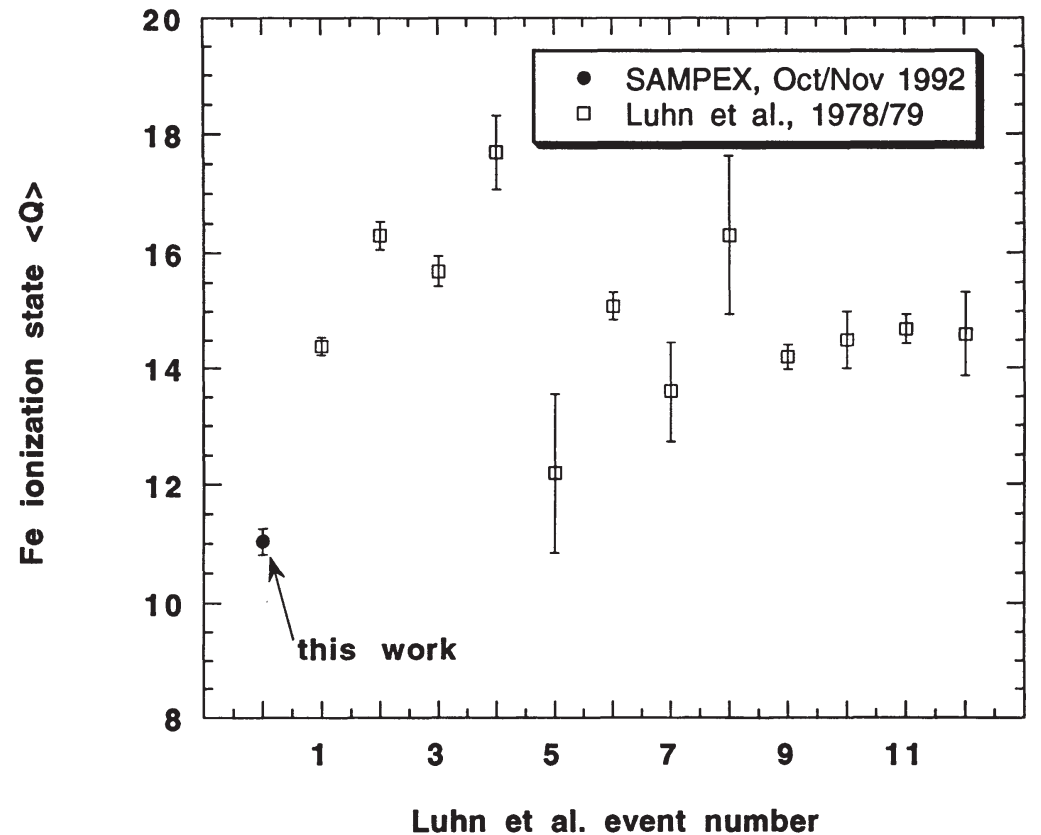

Fig. 11.-Comparison of Fe ionization state deduced here with each of the 12 events in the survey by Luhn et al. (1985) 


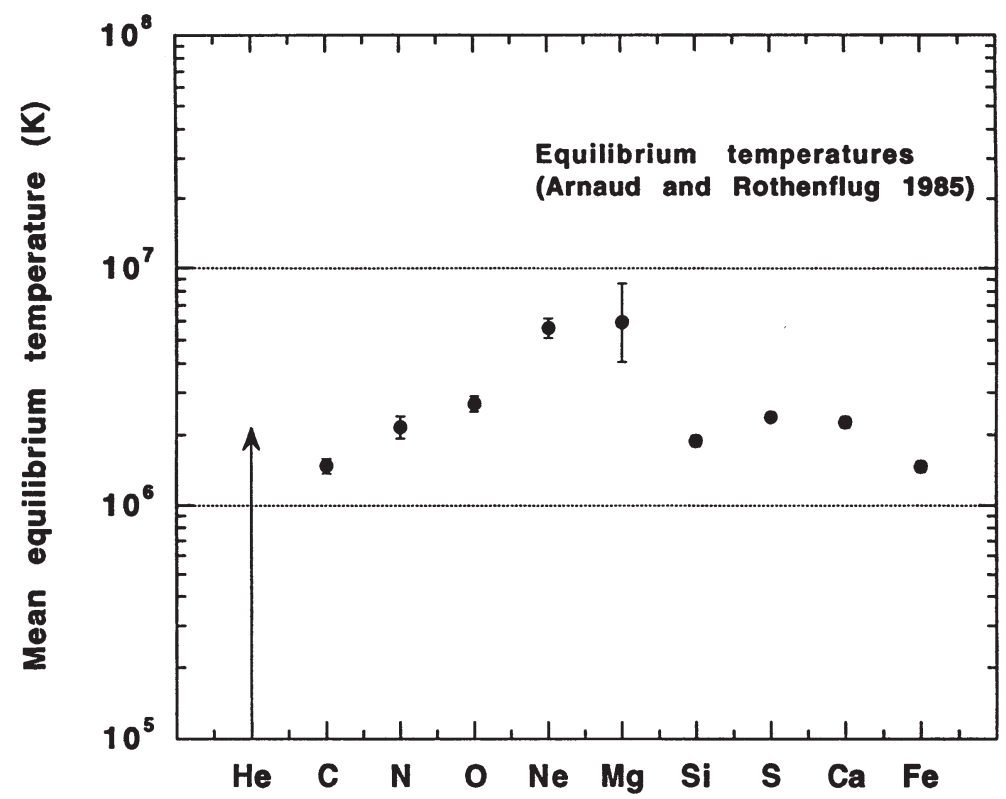

Fig. 12.-Deduced equilibrium ionization temperatures implied by the mean ionization states found in this study using the ionization tables of Arnaud \& Rothenflug (1985). Notice that the implied temperatures for $\mathrm{Ne}$ and $\mathrm{Mg}$, in particular, are much higher than for other elements and that no single temperature yields a good fit to the data.

\& Bame 1968; Bürgi \& Geiss 1986), it has been long recognized that the solar wind observed in interplanetary space is a direct sample of the plasma close to the Sun. When the first direct measurements of solar energetic particle ionization states (Sciambi et al. 1977) showed ionization states closer to those expected from coronal temperatures of a few million $\mathrm{K}$ rather than the 10-20 MK at the flare site, it provided evidence that the energetic particles were accelerated out of coronal material. Subsequent work has accumulated evidence that large solar energetic particle events like that examined here are members of a class of events in which large coronal and interplanetary shocks are the accelerating mechanism for the ions observed at 1 AU (e.g., Mason et al. 1984; Lin 1987; Cane et al. 1988; Gosling 1993). It is therefore appropriate to compare the ionization states derived here with those in the solar wind. We emphasize that the new observations reported here extend the energy range of solar energetic particle (SEP) charge state measurements to significantly higher energies than earlier surveys. We find no energy variations in the ionization states that would give evidence for differing seed populations or processes over the range of measurements reported here. However, in at higher energies $\left(>20 \mathrm{MeV}\right.$ nucleon $\left.^{-1}\right)$ in this same event, Leske et al. (1995) found ionization states for Fe that were close to the earlier results of Luhn et al. (1984).

The direct measurements of solar energetic particle charge states and derivations of equilibrium temperatures in previous work were carried out in the absence of comprehensive measurements of the solar wind ionization states. However, new instrumentation (Gloeckler et al. 1992) launched on the Ulysses deep space probe in 1990 has been systematically surveying the individual charge state distributions of solar wind ions. Surveys published to date (e.g., Ipavich et al. 1992; Galvin et al. 1995; von Steiger \& Geiss 1995) have shown that the ionization states of different elements do not correspond to a single ionization temperature as computed from equilibrium calculations such as those of Arnaud \& Rothenflug (1985). In fact, this lack of correspondence was predicted by the detailed model of Bürgi \& Geiss (1986). Therefore, the lack of a single ionization temperature in Figure 12 does not necessarily rule out agreement with actual solar wind ionization states.

Because of the trajectory of Ulysses, its observations do not sample the solar wind conditions near Earth for a simultaneous comparison with the SAMPEX results presented here. However, Ipavich et al. (1992) carried out a survey of 92 consecutive days in 1990-1991 of in-ecliptic measurements of the $\mathrm{O}$ and $\mathrm{Fe}$ solar wind charge states that can be used here to provide a context for the new SEP charge state measurements. During the survey, solar wind charge distributions were calculated every $\sim 13$ minutes and binned according to the $\mathrm{O}$ temperature as deduced by the $\mathrm{O}^{7+} / \mathrm{O}^{6+}$ ratio. It was found that at higher $\mathrm{O}$ temperatures, the $\mathrm{Fe}$ also showed higher ionization states, as might be expected. However, even at the highest $\mathrm{O}$ temperatures $\left(>2 \times 10^{6} \mathrm{~K}\right)$ the $\mathrm{Fe}$ charge state distribution covered the range from $\mathrm{Fe}^{7+}$ to $\mathrm{Fe}^{16+}$. When we estimate from Figure 4 of Ipavich et al. (1992), we find that the mean $\mathrm{Fe}$ charge state for the highest temperature $\mathrm{O}$ periods was $\sim 12.5$, while at the lower $\mathrm{O}$ temperatures, the mean $\mathrm{Fe}$ charge state was in the range $\sim 10.5-11.5$. These charge states are much closer to the $\mathrm{Fe}$ charge state reported in the present work than to values in the earlier studies that found an average $\mathrm{Fe}$ charge state of $\sim 15$. We believe it is particularly significant that the surveys of Ipavich et al. (1992) and Galvin et al. (1995) did not find any periods in which an average $\mathrm{Fe}$ charge state of $\sim 15$ was typical in the solar wind. Since large solar particle events last for several days, it would be expected, if the energetic particle population is indeed accelerated out of the solar wind by large-scale shocks, that energetic particles would sample average solar wind ionization states and so should be compared with surveys such as those discussed here.

Recently, von Steiger \& Geiss (1995) have presented new surveys of solar wind $\mathrm{Fe}$ charge states from Ulysses. During a fast solar stream period ("coronal holes") they found that the Fe charge state distribution was well fitted by an equilibrium temperature of $1.26 \mathrm{MK}$, close to the $1.47 \mathrm{MK}$ shown here 
(Fig. 12). This agreement may be fortuitous, however, because the limited solar wind data near Earth for the period 1992 October 26-November 2 (from the NSSDC's on-line "Omni" data set) showed a low-speed period during which the solar wind speed declined from approximately $550 \mathrm{~km} \mathrm{~s}^{-1}$ to 400 $\mathrm{km} \mathrm{s}^{-1}$. In a low-speed "interstream period," von Steiger \& Geiss (1995) found that higher Fe charge states were present (up to charge 16); nevertheless, even during this period, there were significant abundances of $\mathrm{Fe}$ charge states down to +8 , and the mean $\mathrm{Fe}$ charge state was $\sim 11.4$, close to the value in Table 1.

While the SEP Fe charge state reported here appears to be close to the values observed in the solar wind, other discrepancies remain. For example, the $\mathrm{O}$ temperature deduced here is somewhat higher than typical values found by Ipavich et al. (1992), and other processes may be influencing the energetic particle ionization states (e.g., Reames, Meyer, \& von
Rosenvinge 1994). In addition, at energies $>20 \mathrm{MeV}$ nucleon $^{-1}$, Leske et al. (1995) found Fe charge states essentially in agreement with the earlier work of Luhn et al. (1984). Clearly, comparison between ionization states of solar wind versus solar energetic particles needs to be done during similar time periods in order to permit more detailed analysis. Instruments on the Wind and Advanced Composition Explorer ( $A C E)$ spacecraft will permit such investigations in the future.

We thank the members of the Space Physics Group at the University of Maryland Department of Physics and the Caltech Space Radiation Laboratory who designed, constructed, and calibrated the LICA and PET instruments; and the Small Explorer Project Office at Goddard Space Flight Center for the construction and launch of the SAMPEX spacecraft. This work was supported by NASA under contract NAS5-30704.
Adams, J. H., Jr., et al. 1993, Adv. Space Res., 13, 367

Arnaud, M., \& Rothenflug, R. 1985, A\&AS, 60, 425

Baker, D. N., Mason, G. M., Figueroa, O., Colon, G., Watzin, J. G., \& Aleman, R. M. 1993, IEEE Trans. Geosci. Remote Sensing, 31, 531

Bürgi, A., \& Geiss, J. 1986, Sol. Phys., 103, 347

Cane, H. V., Reames, D. V., \& von Rosenvinge, T. T. 1988, J. Geophys. Res., 93, 9555

Cook, W. R, et al 1993, IEEE Trans. Geosci. Remote Sens., 31, 565

Cooper, J. F., Guzik, T. G., \& Wefel, J. P. 1993, private communication

Evans, L. C., \& Stone, E. C. 1969, J. Geophys. Res., 74, 5127

Flückiger, E. O., Smart, D. F., \& Shea, M. A. 1986, J. Geophys. Res., 91,7925

Galvin, A. B., Ipavich, F. M., Cohen, C. M. S., Gloeckler, G., \& von Steiger, R. 1995, Space Sci. Rev., 72, 65

Gloeckler, G., et al. 1992, A\&AS, 92, 267

Gloeckler, G., et al. 1994, J. Geophys. Res., 99, 17637

Gloeckler, G., et al. 1981, Proc. 17th Int. Cosmic Ray Conf. (Paris), 3, 136

Gosling, J. T. 1993, J. Geophys. Res., 98, 18937

Hovestadt, D., et al. 1981a, Adv. Space Res., 1, 61

- 1981b, ApJ, 246, L81

Hovestadt, D., \& Meyer, P. 1969, Acta Phys. Acad. Sci. Hungaricae Suppl., 29, 525

Hundhausen, A. J., Gilbert, H. E., \& Bame, S. M. 1968, J. Geophys. Res., 73, 5485

Ipavich, F. M., Galvin, A. B., Geiss, J., Ogilvie, K. W., \& Gliem, F. 1992, in Solar Wind Seven, ed. E. Marsch \& R. Schwenn (COSPAR Colloq. Ser., 3) (Oxford: Pergamon Press), 369

Leske, R. A., Cummings, J. R., Mewaldt, R. A., Stone, E. C., \& von Rosenvinge, T. T. 1995, ApJ, submitted

\section{REFERENCES}

Lin, R. P. 1987, Rev. Geophys., 25, 676

Luhn, A., et al. 1985, Proc. 19th Int. Cosmic Ray Conf. (La Jolla) (NASA CP-2376), 4, 241

Luhn, A., Klecker, B., Hovestadt, D. Gloeckler, G., Ipavich, F. M., Scholer, M., Fan, C. Y., \& Fisk, L. A. 1984, Adv. Space Res., 4, 161

Mason, G. M., Gloeckler, G., \& Hovestadt, D. 1984, ApJ, 280, 902

Mason, G. M., Hamilton, D. C., Walpole, P. H., Heuerman, K. F., James, T. L., Lennard, M. H., \& Mazur, J. E. 1993, IEEE Trans. Geosci. Remote Sensing 31,549

Mcllwain, C. E. 1961, J. Geophys. Res., 66, 368

Möbius, E., Hovestadt, D., Klecker, B., Scholer, M., Gloeckler, G., \& Ipavich, F. M. 1985, Nature, 318, 426

Mullan, D. J., \& Waldron, W. L. 1986, ApJ, 308, L21

Reames, D. V., Meyer, J. P., \& von Rosenvinge, T. T. 1994, ApJS, 90, 649

Rossi, B., \& Olbert, S. 1970, in Introduction to the Physics of Space, ed. L. I. Schiff(New York: McGraw-Hill), 107

Schulz, M., \& McNab, M. C. 1987, Geophys. Res. Lett., 14, 182

Sciambi, R. K., Gloeckler, G., Fan, C. Y., \& Hovestadt, D. 1977, ApJ, 214, 316

Selesnick, R. S., Cummings, A. C., Cummings, J. R., Leske, R. A., Mewaldt R. A., Stone, E. C., \& Rosenvinge, T. T. 1993, ApJ, 418, L45

Shea, M. A., \& Smart, D. F. 1983, Proc. 18th Int. Cosmic Ray Conf (Bangalore), 3, 415

Van Allen, J. A., Fennell, J. F. \& Ness, N. F. 1971, J. Geophys. Res., 76, 4262

von Steiger, R., \& Geiss, J. 1995, in Cosmic Winds and the Heliosphere, ed. J. R. Jokipii, C. P. Sonnett, \& M. S. Giampapa (Tucson: Univ. of Arizona Press), 1 